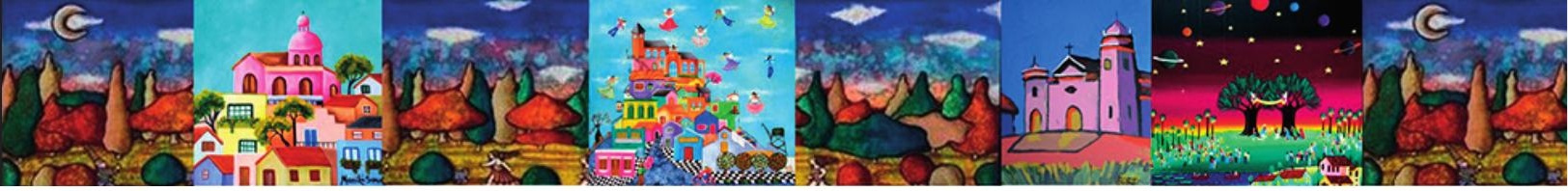

Artículo de Investigación E18A03 * Proyecto "Factores motivacionales de logro y poder y su relación

con la actitud innovadora del individuo”. * Universidad Autónoma de Tamaulipas, Méjico.

Recibido: 10.06.2020 * Aprobado versión final 30.12.2020 * JEL: D20, J24, M12, O30, O31

Pp. 79-96. doi: 10.33571/teuken.v12n18a4

\title{
Factores motivacionales de logro y poder y su relación con la actitud innovadora del individuo.
}

\author{
Motivational factors of achievement and power \\ and its relationship with innovative attitude \\ of the individual.
}

\section{Bernardo Nahuat Román - Magda Lizet Ochoa Hernández Lisette Farah Simón \\ MÉJICO}

Resumen: existe evidencia en la literatura de que la actitud adoptada por los individuos hacia la innovación se ve influida por sus factores intrínsecos; por ello, este trabajo tiene como objetivo determinar la influencia de los factores motivacionales de logro y de poder en la actitud innovadora del individuo. El diseño metodológico adoptó un enfoque cuantitativo de corte transversal con alcance explicativo. Mediante un estudio de caso se recabaron 56 cuestionarios a trabajadores de una empresa mediana en la ciudad de Tampico, Tamaulipa; después se aplicó una regresión múltiple que evidenció la influencia positiva y significativa de los factores motivacionales de logro y de poder en la actitud innovadora del individuo. Los resultados amplían la comprensión de la relación entre los factores motivacionales y la actitud innovadora del individuo.

Palabras clave: individuo; actitud innovadora; factor motivacional de logro; factor motivacional de poder; factores intrínsecos.

Abstract: There is evidence in the literature that its intrinsic factors influence the attitude adopted by individuals towards innovation. Therefore, this work aims to determine the influence of motivational factors of achievement and power on the innovative attitude of the individual. The methodological design adopted a cross-sectional quantitative approach with explanatory scope. This work collects 56 questionnaires from workers in a medium-sized company in Tampico, Tamaulipas, through a case study. It applies a multiple regression method that showed the positive and significant influence of motivational factors of achievement and power on the innovative attitude of the individual. The results broaden the understanding of the relationship between motivational factors and the innovative attitude of the individual.

Keywords: Individual; innovative attitude; motivational factor of achievement; motivational factor of power; intrinsic factors.

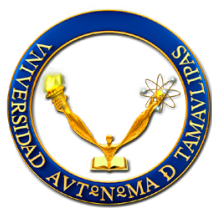

Bernardo Nahuat Román es estudiante del Doctorado en Gestión Estratégica de Negocios, reconocido en el Programa Nacional de Posgrados de Calidad (PNPC) CONACYT; Maestro en Administración con Especialidad en Finanzas; Contador Público y Auditor. Universidad Autónoma de Tamaulipas. Facultad de Comercio y Administración de Tampico. 


\title{
Fatores motivacionais de conquista e poder e sua relação com a atitude inovadora do indivíduo.
}

\begin{abstract}
Resumo: existem evidências na literatura de que a atitude adotada pelos indivíduos em relação à inovação é influenciada por seus fatores intrínsecos. Portanto, este trabalho tem como objetivo determinar a influência de fatores motivacionais de conquista e poder na atitude inovadora do indivíduo. O desenho metodológico adotou uma abordagem quantitativa transversal com escopo explicativo. Por meio de um estudo de caso, 56 questionários foram coletados de trabalhadores de uma empresa de médio porte na cidade de Tampico, Tamaulipas, para posteriormente aplicar uma regressão múltipla que mostrou a influência positiva e significativa de fatores motivacionais de conquista e poder sobre a atitude inovadora da empresa. Individual. Os resultados ampliam a compreensão da relação entre fatores motivacionais e a atitude inovadora do indivíduo.
\end{abstract}

Palavras-chave: indivíduo; atitude inovadora; fator motivacional de conquista; fator de poder motivacional; fatores intrínsecos

\section{Introducción}

a importancia de la innovación es indudable tanto para la academia como para el mundo de los negocios. Autores como Koudelková y Milichovsky (2015) señalan que es uno de los más importantes factores para el crecimiento de las empresas y otros como Varadarajan y Jayachandran (1999) mencionan que se encuentra íntimamente ligada al éxito empresarial y a la ventaja competitiva; en ese mismo sentido se pueden encontrar en la literatura numerosas afirmaciones, incluso Schumpeter (1934) es una referencia frecuente al respecto. Sin embargo, para entenderla mejor se hace necesario profundizar en su nivel básico, es decir, aquella que nace de la actitud innovadora del individuo y determinar el efecto de sus factores intrínsecos, ya que a decir de McCabe (2002) la innovación está relacionada con la actuación de los individuos y las metas que estos persigan estarán ligadas a las razones para comprometerse con sus tareas (Lai, 2011).

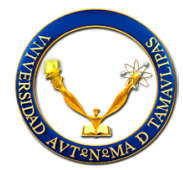

\footnotetext{
Magda Lizet Ochoa Hernández es Doctora en Nuevas Tendencias en Dirección de Empresas y Profesora investigadora de tiempo completo en la Universidad Autónoma de Tamaulipas, Facultad de Comercio y Administración de Tampico.

Contacto: mlochoa@docentes.uat.edu.mx
}

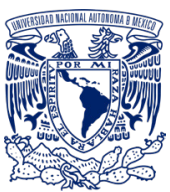

\footnotetext{
Lisette Farah Simón es Doctora en Ciencias de la Administración, Maestra en Ingeniería Mecánica. Ingeniera Industrial y Profesora investigadora de tiempo completo en la Universidad Nacional Autónoma de México. Facultad de Contaduría y Administración.

Contacto: Ifarah@live.com.mx
} 
Los individuos con sus motivaciones intrínsecas son la base de toda empresa y con la innovación que estos puedan generar juegan un papel clave en las organizaciones (Bettencourt et al., 2017; Jong y Hartog, 2008); así pues, en la medida en que se logre identificar la influencia de estos factores motivacionales intrínsecos se podría impulsar la actitud innovadora del individuo y por consiguiente la innovación organizacional. En esta línea, Kunz y Linder (2015) en su estudio mencionan lo poco que se sabe acerca de los factores intrínsecos en los individuos, que están relacionados con su intención de involucrarse en la innovación y con la forma en que estas características afecta al conjunto de la empresa.

Los mismos autores señalan que los factores motivacionales de tipo psicológico también juegan un rol clave, aunque existen condiciones laborales en el contexto del individuo que influyen en su intención de innovar, tales como la cultura organizacional, el tamaño de la empresa, el mercado en el que esta se desenvuelve, la centralización de autoridad, entre otras (Kunz y Linder, 2015).

La motivación es un factor intrínseco que se relaciona con las razones ocultas detrás del comportamiento de las personas (Lai, 2011), y tales razones pueden ser sus motivaciones de logro y de poder; estas dos, junto con otra llamada de afiliación, son conocidas como la teoría de las necesidades de McClelland (1961). Esta teoría básicamente pretende explicar cómo las necesidades de logro, poder y afiliación influyen en las acciones de los individuos en un ambiente empresarial (McClelland, 1961) y puede encontrarse en la literatura también con el nombre de teoría de las tres necesidades, teoría de las necesidades adquiridas, teoría de las necesidades motivacionales o teoría de las necesidades aprendidas.

Las aproximaciones previas que han abordado la innovación a nivel del individuo en un ambiente empresarial se han enfocado en los gerentes o agentes externos, de los que se presume que por la naturaleza de sus puestos ejercen una mayor influencia en las organizaciones, lo que deja de lado al grueso de los trabajadores que se ubican en un nivel inferior de la estructura jerárquica (Kunz y Linder, 2015); por lo anterior, se hace interesante estudiar al individuo sin discriminarlo por su posición en la empresa.

A partir de la consideración de que existe todavía un vacío en la conceptualización y la medición de los factores motivacionales que están relacionados con la innovación (Shalley et al., 2004), y de que pocos estudios han abordado los detonantes para generar innovación a nivel del individuo (Bammens, 2015), este trabajo tiene como objetivo determinar la influencia de las motivaciones de logro y de poder en la actitud innovadora del individuo. Si bien la academia ha teorizado sobre la 
innovación a nivel del individuo (Bammens, 2015), la presente propuesta resulta original al realizar un estudio de caso en una empresa dedicada al comercio, sector en el que a juicio de autores como Pérez et al. (2014) hay poca innovación.

El trabajo se presenta estructurado en cuatro apartados. En el primero se presenta una revisión de la literatura sobre los conceptos teóricos de la relación de los factores motivacionales y la actitud innovadora del individuo. En el segundo apartado se describe el diseño metodológico, seguido de los resultados y la discusión que se presentan en el tercer apartado y, por último, se presentan las conclusiones, limitaciones, contribuciones, así como futuras líneas de investigación.

\section{Revisión de la literatura}

Estudios en psicología han dejado evidencia de que las características de los individuos inmersas en su personalidad juegan un papel crucial en su conducta y su actitud hacia la toma de decisiones (Batra y Vohra, 2016). La importancia de la motivación como determinante en gran medida de lo que los individuos realizan, rastreada en estudios como el de Bettencourt et al. (2017), es comprensible si se parte de que los objetivos de un individuo están relacionados con sus razones para comprometerse con ellos (Lai, 2011).

Diversos autores han conceptualizado la motivación; por ejemplo Lahey (1999) la define como "un estado interno que activa y dirige nuestros pensamientos" (p. 411). Para Davidoff (1989) es "un estado interno que puede ser el resultado de una necesidad, y se le caracteriza como algo que activa o excita la conducta que por lo común se dirige a la satisfacción del requerimiento instigador". (p. 331)

Lai (2011) señala que la motivación está relacionada con las razones ocultas detrás del comportamiento de las personas y se clasifica en extrínseca e intrínseca; esta última se puede entender como "aquella que trae, pone, ejecuta, activa el individuo por sí mismo cuando lo desea, para aquello que le apetece" (Soriano, 2001, p. 7). Dentro de las motivaciones intrínsecas se puede mencionar a las motivaciones de logro y de poder.

La motivación de logro se puede definir como la que lleva al individuo a tomar y solventar de forma exitosa un reto u objetivo por el que obtendrá reconocimiento ante la sociedad (Soriano, 2001). Para Monroy y Rodríguez (2012) la motivación "es el deseo de sobresalir, de superar unas metas o a otras personas" (p. 7) y Lahey (1999) la entiende como "la necesidad psicológica de alcanzar el éxito en los estudios, el deporte, la profesión u otra situación de competencia" (p. 423). Así pues, los individuos que 
posean este tipo de motivación encontrarán interesantes los retos u obstáculos que se les presente en su trabajo diario y se comprometerán consigo mismos en una búsqueda de cómo solventarlos.

Ahora bien, para abordar la motivación de poder es conveniente primero entender al concepto de poder. Hardy (1996) señala que se lo ha relacionado con el movimiento de recursos frecuentemente, así como con la connotación negativa de poder sobre otra persona. La misma autora agrega que sin poder sería difícil llevar a cabo cambios estratégicos en los negocios. Clegg et al. (2006) se refieren al poder en un sentido positivo con el que se pueden lograr grandes cosas, tales como: hacer relaciones, democracias, libertad, formar capacidades, decisiones de cambio, y además puede impulsar y frenar las cosas.

Específicamente, la motivación de poder "se refiere a hacer que otros se comporten de una forma que habitualmente no se comportarían" (Monroy, 2012, p. 7), es decir influir o manipular el comportamiento de los compañeros de trabajo si se habla en el contexto de la empresa. Cuando surgen obstáculos o retos, las personas que tienen esta motivación harán mover a los demás en busca de una solución, la que puede ser simplemente encauzar a las personas a realizar sugerencias.

Ambas motivaciones (de logro y de poder) son parte del perfil psicológico de los individuos y se reflejarán en la conducta innovadora que adopten. En este sentido, autores como Goepel et al. (2012) y Xu (2011) coinciden en la importancia del perfil psicológico de los individuos para generar innovaciones exitosas.

Para Batra y Vohra (2016), "la innovación individual requiere la voluntad de las personas para experimentar con nuevos productos, procesos, métodos, tareas y tecnologías" (p. 769); McCabe (2002) por su parte, señala que la innovación está relacionada con la actuación de los individuos y las metas que estos persigan. En consideración de lo anterior y siguiendo a Goepel et al. (2012), este trabajo entiende la actitud innovadora del individuo como la disposición que este tiene para introducir, adoptar o llevar a la práctica ideas nuevas.

En la literatura se utilizan diferentes términos para aludir a esa actitud, tales como: conducta del empleado innovadora (Janssen, 2005), conducta innovadora en el lugar de trabajo (Yuan y Woodman, 2010), innovación personal (Agarwal y Prasad, 1998), innovación individual (Batra y Vohra, 2016; Goepel et al., 2012) conducta de trabajo innovadora (Jong y Hartog, 2008), conducta innovadora individual (Kleysen y Street, 2001), entre otros; pero, en realidad son conceptos similares. Autores como Batra y Vohra (2016) coinciden en que en la literatura se puede encontrar de 
forma indistinta como innovación individual, innovación personal o disposición personal a la innovación.

El estudio de la innovación al nivel del individuo todavía se encuentra en una etapa de desarrollo; aunque las aproximaciones pioneras existentes en esta vertiente han propuesto algunas explicaciones (Yuan y Woodman, 2010), debido a su escasez todavía no logran abordar las múltiples razones por las que un individuo tiende a prácticas relacionadas con la innovación (Shalley et al., 2004). En la medida en que se quiera entender la actitud innovadora del individuo, la evidencia sugiere que se debe seguir indagando en lo que lo mueve y lo hace proclive a innovar.

En este sentido, Cano et al. (2014) señalan que los estudios empíricos de forma común lo que más utilizan como unidad de análisis es la empresa, un conjunto de empresas de diversos sectores o empresas de la misma industria. Bettencourt et al. (2017) coinciden y a esta lista agregan los equipos de trabajo, lo que muestra el vacío en el conocimiento ya que la literatura nunca se ha enfatizado en investigaciones empíricas a nivel del individuo (Mura y Longo, 2013), a pesar de que de este emana el conocimiento (Grant, 1996a, 1996b) y de que la innovación que puedan generar los individuos es fundamental para que una empresa pueda tener éxito (Birdi et al., 2016).

Como señalan Kunz y Linder (2015), investigaciones previas se enfocaron en gerentes o mandos, de los que por su posición en la estructura jerárquica se podría presumir una mayor influencia en las organizaciones; sin embargo, con el paso del tiempo, al grueso de los trabajadores se le ha estado quitando esa etiqueta de agentes pasivos que solo recibe órdenes, pues se entiende que ellos pueden fungir como expertos en sus áreas o promotores de cambios de procesos diarios. Autores como Zhou y George (2001) confirman el rol clave de los trabajadores al momento de desarrollarse o implementarse algún cambio organizacional. Basadur (2004) va más allá al afirmar que son precisamente las personas quienes detectan áreas de oportunidad, y de ahí nacen las ideas que a la postre se transforman en cosas nuevas.

Dicho de otra forma, son los individuos quienes al abordar problemas cotidianos desde diferentes ángulos se convierten en generadores de buenas ideas (Jong y Hartog, 2008), las que por provenir directamente del área de trabajo van enfocadas en soluciones o mejoras prácticas, por lo que se justifica considerar las percepciones del grueso de los trabajadores.

Aunque ha habido aproximaciones previas en la academia que buscan identificar lo que mueve al individuo a innovar, las propuestas realizadas a través de modelos integrales son escasas y no tienen el sustento empírico 
necesario (Bettencourt et al., 2017); razón por lo cual se hace necesario profundizar en el tema.

\section{Hipótesis}

McClelland (1961) señala que las personas con alta motivación de logro se sienten atraídos por tomar y completar objetivos que les signifiquen un alto grado de desafío, aun con los riesgos inherentes para conseguirlos; una persona con esta motivación será persistente, buscará oportunidades y asumirá riesgos calculados, lo que puede ser preámbulo de innovación. En esa línea, Gatewood et al. (1995) coinciden en que la principal característica de un individuo con la motivación de logro es mantenerse orientado hacia altos desafíos y en busca de oportunidades para probarse; con esto se puede suponer que una forma sencilla será desafiando, cuestionando o modificando las cosas rutinarias o aquellas que presenten algún tipo de reto.

Kunz y Linder (2015), con base en su estudio empírico con la técnica de viñeta y cuya unidad de análisis fueron 84 estudiantes recién graduados en Dinamarca, encuentran que a mayor grado de la necesidad de logro mayor es la intención de innovar en las prácticas gerenciales. Por su lado, los estudios inferenciales de Winter (2010) y Cho y Pucik (2010) citados por Larawan (2011) dejaron evidencia de la íntima relación entre la motivación de logro con la innovación en dirección del éxito empresarial. Con base en lo anterior, se propone la primera hipótesis de esta investigación.

Hipótesis 1. Los factores motivacionales de logro tienen una influencia positiva en la actitud innovadora del individuo.

Ahora bien, en las organizaciones continuamente se encuentran reglas o prácticas de trabajo organizacionales ya establecidas, mismas que aunque no sean las mejores llevan tiempo inmersas en la cotidianidad; por ello, una forma de ejercer o lograr más poder será sin duda ser disruptivo, alterar el status quo. En este sentido, McClelland (1961) plantea que al individuo con alta motivación de poder le gusta ser competitivo y ganador, por ello buscará ser persuasivo y construirá redes de apoyo para solucionar obstáculos que se le presenten. Valera (2008) define al diseñador de innovaciones como aquel individuo que utiliza sus redes de contacto (las personas sobre las que ejerce un nivel de poder y en su búsqueda de más poder y reconocimiento) para dirigir sus esfuerzos en orientar las actividades de la organización, en busca de un valor agregado.

Por su parte, McClelland y Burnham (2003) señalan que las personas con la motivación de poder sienten el deseo de hacer las cosas mejor o más eficientemente que antes, para esto se presume que tendrían que llevar 
a cabo las cosas de una forma diferente si realmente buscan mejorarlas. En sentido inverso, Kunz y Linder (2015) no encuentran evidencia de que a mayor grado de la necesidad de poder sea mayor la intención para innovar prácticas gerenciales. Por lo anterior, se propone la siguiente hipótesis de investigación:

Hipótesis 2. Los factores motivacionales de poder tienen una influencia positiva y significativa en la actitud innovadora del individuo.

Figura 1. Modelo conceptual propuesto.

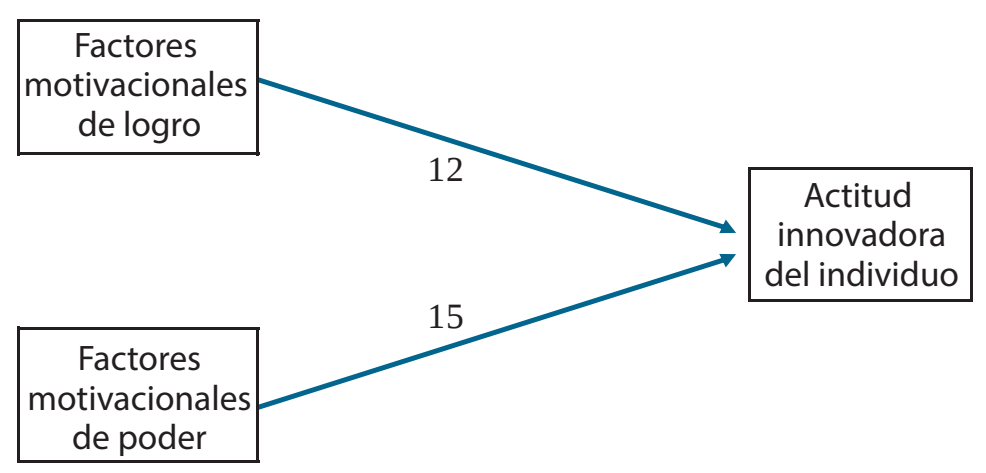

Fuente: elaboración propia

\section{Diseño Metodológico}

Para cumplir con su objetivo, la investigación adoptó un enfoque cuantitativo de corte transversal con alcance explicativo. Mediante un estudio de caso se seleccionó una empresa mediana dedicada a la comercialización de ropa en Tampico, Tamaulipas. Este tipo de empresas se caracteriza porque no presentan innovación tecnológica; sin embargo, por enfocarse en la moda, requieren estar continuamente reinventándose.

Para empezar, a principios del mes de febrero de 2019 se llevaron a cabo dos entrevistas semi-estructuradas (con el gerente comercial y el empleado con mayor antigüedad) en la empresa. Entre lo manifestado se destaca que sus integrantes presentan una combinación sui generis de conocimientos y experiencia, así como también que la búsqueda constante de innovación organizacional es parte prioritaria de su modelo de negocio, buscando siempre la excelencia en el servicio y la optimización de recursos (tiempo y dinero). Si se parte de que el conocimiento es la antesala de la innovación (Buenechea-Elberdin et al., 2017) o, dicho de otra forma, que para crear conocimiento nuevo se necesita de un conocimiento previo (Buenechea-Elberdin et al., 2018), desde esta fase se sugiere la existencia de innovación en la empresa. 
La unidad de análisis se definió como el total de los empleados de la empresa que suman 68 entre el área operativa y áreas habilitadoras. Como instrumento de recolección de datos se utilizó el cuestionario de Kunz y Linder (2015), en virtud de que se ajusta al propósito de la investigación. En total, el cuestionario quedó compuesto por 16 reactivos (4 ítems de factores motivacionales de logro, 3 ítems de factores motivacionales de poder y 9 ítems de actitud innovadora del individuo). Todos los reactivos fueron basados en percepciones y medidos con una escala Likert donde 1 significa totalmente en desacuerdo y 5 significa totalmente de acuerdo.

El trabajo de campo se llevó a cabo en febrero de 2019, lográndose recabar 58 cuestionarios utilizables los cuales se concentraron en un archivo de Excel, para posteriormente ingresarlos al software Statistical Package for the Social Sciences (SPSS por sus siglas en inglés) versión 21 y generar estadística descriptiva.

Tabla 1. Datos estadísticos de las personas encuestadas

\begin{tabular}{|c|c|c|}
\hline & $\mathbf{N}$ & $\%$ \\
\hline \multicolumn{3}{|l|}{ Género } \\
\hline Mujer & 40 & 71.42 \\
\hline Hombre & 16 & 28.58 \\
\hline Total & 56 & \\
\hline \multicolumn{3}{|l|}{ Edad } \\
\hline$<20$ años & 1 & 01.78 \\
\hline Entre 20 a 25 años & 19 & 33.92 \\
\hline$>25$ años & 36 & 64.30 \\
\hline Total & 56 & \\
\hline \multicolumn{3}{|l|}{ Grado de estudios } \\
\hline $\begin{array}{l}\text { Preparatoria o licenciatura } \\
\text { trunca }\end{array}$ & 18 & 32.14 \\
\hline Estudiante de licenciatura & 13 & 23.21 \\
\hline Licenciatura & 21 & 37.50 \\
\hline Maestría & 4 & 07.15 \\
\hline Total & 56 & \\
\hline \multicolumn{3}{|l|}{ Área de trabajo } \\
\hline Operaciones & 30 & 53.57 \\
\hline Staff & 26 & 46.43 \\
\hline Total & 56 & \\
\hline
\end{tabular}

Fuente: Elaboración propia en base tablas de frecuencia del SPSS. 
El tratamiento de la información se hizo mediante una regresión múltiple; esta técnica es ideal, ya que permite determinar la relación entre un conjunto de variables independientes y una variable dependiente mediante un modelo lineal (Montero, 2016). Para identificar el efecto de las variables factores motivacionales de logro y factores motivacionales de poder en la actitud innovadora del individuo $(\mathrm{H} 1$ y H2), se propuso el siguiente modelo:

$$
\mathrm{AII}=\beta_{0}+\beta_{1} \text { Faclog }+\beta_{2} \text { Facpod }+\varepsilon_{i}
$$

Donde:

All= Actitud innovadora del individuo.

Faclog=factor motivacional de logro.

Facpod=factor motivacional de poder.

$\varepsilon_{i}=$ error (aquello que el modelo no puede explicar).

\section{Resultados}

En una primera instancia se procedió a la exploración de los datos recabados, asegurando que no se presentaran valores ausentes (missing values en inglés). En lo referente a valores atípicos o anómalos (outliers en inglés), se encontraron dos cuestionarios con valores incongruentes con el resto de los datos recabados, por lo que se decidió eliminarlos de la encuesta para evitar que distorsionaran los resultados de la investigación (Kline, 2015), lo que dejó 56 cuestionarios para estudio (ver Tabla 1).

En una segunda instancia se procedió a analizar la estadística descriptiva (ver Tabla 2); destaca la actitud innovadora del individuo por sus valores superiores, tanto en el mínimo como en la media, así como por su desviación estándar, pues la mayor parte de los datos se agrupan mejor alrededor de la media en comparación con las otras 2 variables.

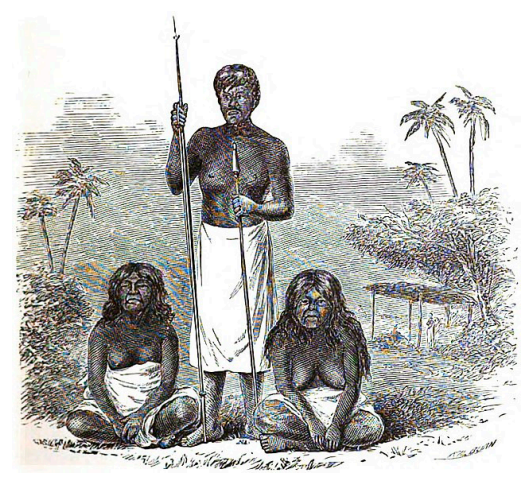


Tabla 2. Estadística descriptiva de las variables.

\begin{tabular}{|l|c|c|c|c|c|}
\hline \multicolumn{2}{|c}{ Variable } & Mínimo & Máximo & Media $\begin{array}{r}\text { Desviación } \\
\text { estándar }\end{array}$ \\
\hline $\begin{array}{l}\text { Factor } \\
\text { motivacional } \\
\text { de logro }\end{array}$ & 56 & 2.25 & 5 & 4.3304 & .54794 \\
\hline $\begin{array}{l}\text { Factor } \\
\text { motivacional } \\
\text { de poder }\end{array}$ & 56 & 2.33 & 5 & 4.2440 & .64976 \\
\hline $\begin{array}{l}\text { Actitud } \\
\text { innovadora } \\
\text { del individuo }\end{array}$ & 56 & 3.22 & 5 & 4.3690 & .37552 \\
\hline
\end{tabular}

Fuente: SPSS con datos recabados.

Para proceder con la regresión es necesario primero validar el modelo, es decir, si cumple con los supuestos de linealidad, independencia de los errores residuales, homocedasticidad, normalidad y no multicolinealidad (Baños et al., 2019). A continuación, se describe como se abordó cada supuesto:

- Supuesto de linealidad: consiste en asegurarse de que la relación entre las variables sea lineal; esto se validó en los diagramas de dispersión parcial.

- Supuesto de independencia de los errores: consiste en que los errores de medición de las variables exógenas tengan independencia entre ellos, por lo que se validó mediante el estadístico de Durbin-Watson; el resultado fue 2,00, cumpliendo con el rango de este test que es de 1.5 a 2.5 (Baños et al., 2019), por lo que se puede asumir que hay independencia de los errores.

- Supuesto de homocedasticidad: hace referencia a que los errores presenten una varianza constante. Esto se validó en los gráficos de dispersión, al encontrarse que la variación de los residuos es uniforme.

- Supuesto de normalidad: por ser igual o más de 50 datos se utilizó la prueba de Kolgomorov, de la que se obtuvo como resultado 0.656; al ser el p valor (sig) >.05 se acepta la hipótesis nula que consiste en que la variable dependiente tiene una distribución normal.

- Supuesto de no multicolinealidad: consiste en asegurarse de que las variables exógenas no están correlacionadas entre sí. Para esto se utilizaron los estadísticos de nivel de tolerancia y los factores de la inflación de la varianza (FIV); cuando los valores de tolerancia son > 0.10 y los FIV $<10$ se asume que no existe multicolinealidad (Baños et 
al., 2019). En este caso, el resultado la tolerancia fue de .973 y los FIV fue de 1.028 por lo que fue solventada la prueba.

A continuación, en la Tabla 3 se hace referencia a la prueba de validez interna por variable mediante el Alfa de Cronbach; como se puede observar, la variable mejor integrada por sus reactivos, es decir, con una alta consistencia interna, es el factor motivacional de logro con un resultado de $.708 \mathrm{y}$, en sentido inverso, la variable factor motivacional de poder es la que menos agrupa sus ítems. Las tres variables se ubican por encima del nivel crítico de 0.6 , lo que indica una consistencia interna apenas aceptable de acuerdo con Fernandes (2010).

Tabla 3. Coeficientes alfa de Cronbach por dimensión

\begin{tabular}{|l|c|}
\multicolumn{1}{|c|}{ DIMENSIONES } & ALFA DE CRONBACH \\
\hline Factor motivacional de logro & .708 \\
\hline Factor motivacional de poder & .610 \\
\hline Actitud innovadora del individuo & .611 \\
\hline
\end{tabular}

Fuente: Elaboración propia.

Al analizar los resultados de la regresión múltiple, se puede ver el impacto positivo y significativo de la motivación de logro $(\beta=0.319, p<0.05)$ y de la motivación de poder $(\beta=0.422, p<0.05)$ sobre la actitud innovadora del individuo propuestas en esta investigación, con lo que se pueden soportar las $\mathrm{H} 1$ y H2.

Mediante la $\mathrm{R}^{2}$ ajustada se puede ver que las dos variables independientes explican en un $29.80 \%$ la variable explicada; de acuerdo con autores como a Hair et al., (2017)by Hair, Hult, Ringle, and Sarstedt, provides a concise yet very practical guide to understanding and using PLS structural equation modeling (PLS-SEM, los valores se pueden considerar al 25\% como débil, al $50 \%$ como moderada y al $75 \%$ como de un efecto sustancial, por lo en este caso se puede considerar entre débil y moderada.

\section{Discusión}

De acuerdo con los resultados, los factores motivacionales de logro y de poder tienen una influencia positiva y significativa sobre la actitud innovadora del individuo, lo que se alinea con la literatura que muestra que las actitudes y la innovación están íntimamente ligadas (Basadur, 2004) y que la motivación es preludio de las actitudes (Koudelková y Milichovsky, 2015). 
El factor motivacional de logro pesa en el individuo al momento de enfrentar situaciones que se puedan considerar obstáculos o que pudieran ser sujetos de mejora. Este tipo de motivación lo empuja a tomar el reto de esforzarse, encontrar una propuesta de solución y llevarlo a un término exitoso, en el que quede solventado. En el mismo sentido, en una investigación experimental, McClelland (1989) encontró que los individuos con una alta necesidad de logro se mostraron más propensos para encontrar nuevas formas de hacer las cosas evitando la rutina.

Si se considera también que la motivación de logro es una determinante en la ambición comercial (McClelland,1961) y que la innovación tiene incidencia en la intencionalidad comercial (Gurol y Atsan, 2006), los individuos con estas características serán clave en las empresas para alcanzar los objetivos organizacionales.

De otro lado, la motivación de poder se puede reflejar en la cotidianidad, cuando los individuos enfrentan situaciones adversas u obstáculos en su labor diaria y tratan de influir en sus compañeros para construir una idea y proponer posibles soluciones, que permitan hacer las cosas de un modo diferente. El simple hecho de que una persona identifique un problema (que no había sido identificado) deja ver que la motivación de poder en el individuo influye para hacer diferente las cosas.

Los resultados son relevantes (aunque no concluyentes) si se considera que este estudio fue llevado a cabo en un escenario real, con individuos laborando en una empresa en operación, a diferencia de estudios como el de Kunz y Linder (2013) que muestran la influencia de las necesidades de poder y logro sobre la innovación tomando como unidad de análisis a estudiantes, que la mayor parte de su tiempo la pasan en aulas y en un entorno de laboratorio, como los mismos autores reconocen.

Así pues, se entiende que el comportamiento de un individuo con respecto a una situación estará determinado en buena parte por su actitud hacia esa situación (Bagozzi, 1981; Fishbein y Ajzen, 1975); es decir, la diferencia entre individuos en cómo encarar una nueva idea o práctica estará basada en su actitud (Rogers, 2003).

En línea con Lo (2014) la evidencia sugiere que las actitudes son un predictor de los individuos hacia la innovación y, si la innovación para las empresas significa ventaja competitiva y éxito empresarial (Varadarajan y Jayachandran, 1999), se puede dimensionar la importancia del presente estudio. 


\section{Conclusiones}

Esta investigación tuvo como objetivo determinar la influencia de los factores motivacionales de logro y de poder en la actitud innovadora del individuo; se confirma que efectivamente los factores motivacionales de logro y de poder sí influyen de forma positiva y significativa en la actitud innovadora del individuo, lo que se alinea con lo señalado en la literatura. De forma complementaria, la investigación sugiere que en negocios dedicados al comercio sí se genera innovación.

La primera aportación de este trabajo va en el sentido académico, ya que estos resultados abonan al conocimiento sobre los factores motivacionales que influyen en la actitud innovadora del individuo. La innovación es cuestión de los seres humanos, no de las empresas, es decir, parte del individuo; por lo que, si se sabe lo que mueve a las personas a innovar, se podría poner las bases para futuros estudios a nivel organizacional.

Una segunda aportación va en el sentido del mundo de los negocios: si las empresas pudieran conocer en qué medida su personal tiene arraigados los factores motivacionales de logro y de poder, podrían gestionarlos mediante talleres, cursos o conversaciones. Ahora bien, para futuras contrataciones, las organizaciones podrían hacer pruebas que filtraran individuos con estos factores motivacionales.

Un punto que podría considerarse sesgo es que la organización que se consideró para el estudio de caso está consciente del concepto y valor de la innovación y la busca explícitamente a través de sus empleados. La mayor limitante de este trabajo es que se trata de un solo estudio de caso por lo que los resultados no se podrían generalizar. Por lo anterior, se sugiere para futuras aproximaciones abordar otras empresas del mismo giro y poder contrastar resultados.

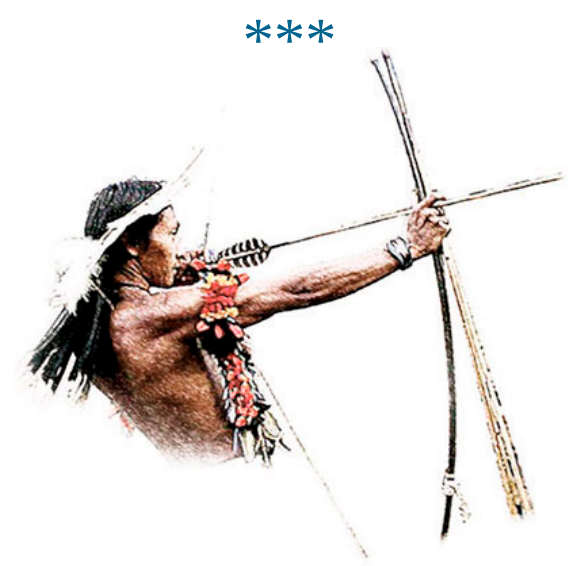




\section{Referencias bibliográficas}

1. Agarwal, R. \& Prasad, J. (1998). A conceptual and operational definition of personal innovativeness in the domain of information technology. Information systems research, 9(2), 204-215.

2. Bagozzi, R. (1981). Attitudes, Intentions, and Behavior: A Test of Some Key Hypotheses. Journal of personality and social psychology, 41(4), 607-627. https://doi.org/10.1037/0022-3514.41.4.607

3. Bammens, Y. (2015). Employees' Innovative Behavior in Social Context: A Closer Examination of the Role of Organizational Care. Journal of Product Innovation Management, 33(3), 244-259. https://doi.org/10.1111/jpim.12267

4. Baños, R. V., Torrado-Fonseca, M. \& Álvarez, M. R. (2019). Anàlisi de regressió lineal múltiple amb SPSS: un exemple pràctic. REIRE Revista d'Innovació $i$ Recerca en Educació, 12(2), 1-10. https://doi.org/10.1344/reire2019.12.222704

5. Basadur, M. (2004). Leading others to think innovatively together: Creative leadership. The Leadership Quarterly, 15(1), 103-121. https://doi.org/10.1016/j. leaqua.2003.12.007

6. Batra, S. \& Vohra, N. (2016). Exploring the linkages of cognitive style and individual innovativeness. Management Research Review, 39(7), 768-785. https://doi.org/10.1108/MRR-03-2014-0047

7. Bettencourt, L. A., Bond III, E. U., Cole, M. S. \& Houston, M. B. (2017). DomainRelevant Commitment and Individual Technical Innovation Performance. Journal of Product Innovation Management, 34(2), 159-180. https://doi. org/10.1111/jpim.12339

8. Birdi, K.; Leach, D. \& Magadley, W. (2016). The relationship of individual capabilities and environmental support with different facets of designers' innovative behavior. Journal of Product Innovation Management, 33(1), 19-35. https://doi.org/10.1111/jpim.12250

9. Buenechea-Elberdin, M., Sáenz, J. \& Kianto, A. (2017). Exploring the role of human capital, renewal capital and entrepreneurial capital in innovation performance in high-tech and low-tech firms. Knowledge Management Research \& Practice, 15(3), 369-379. https://doi.org/10.1057/s41275-0170069-3

10. Buenechea-Elberdin, M.; Kianto, A. \& Sáenz, J. (2018). Intellectual capital drivers of product and managerial innovation in high-tech and low-tech firms. $R \& D$ Management, 48(3), 290-307. https://doi.org/10.1111/radm.12271

11. Cano, M. D. C.; Sánchez, G.C.; González, M. y Pérez, J.C. (2014). El rol del capital intelectual en la innovación de las empresas. European Scientific Journal, 10(28), 348-366. 
12. Clegg, S.; Couparsson, D. \& Phillips, N. (2006). Power and Organizations. Sage publications.

13. Davidoff, L. (1989). Introducción a la Psicología (3a ed.). McGraw-Hill Interamericana.

14. Fernandes, R. (2010). La influencia del capital intelectual en la Innovación de productos: una aplicación a pequeñas y medianas empresas innovadoras de Portugal. [Tesis doctoral, Universidad de Vigo].

15. Fishbein, M. \& Ajzen, I. (1975). Belief, Attitude, Intention, and Behavior: An introduction to Theory and Research. Addison-Wesley publishing company.

16. Gatewood, E. J., Shaver, K. G. \& Gartner, W. B. (1995). A longitudinal study of cognitive factors influencing start-up behaviors and success at venture creation. Journal of business venturing, 10(5), 371-391.

17. Goepel, M.; Hölzle, K. \& zu Knyphausen-Aufseß, D. (2012). Individuals' innovation response behaviour: A framework of antecedents and opportunities for future research. Creativity and Innovation Management, 21(4), 412-426.

18. Grant, R. M. (1996a). Prospering in dynamically-competitive environments: Organizational capability as knowledge integration. Organization science, 7(4), 375-387.

19. Grant, R. M. (1996b). Toward a knowledge-based theory of the firm. Strategic management journal, 17(S2), 109-122.

20. Gurol, Y. \& Atsan, N. (2006). Entrepreneurial characteristics amongst university students: Some insights for entrepreneurship education and training in Turkey. Training, 48(1), 25-38.

21. Hair, J., Hult, T., Ringle, C. \& Sartedt, M. (2017). A primer on partial least squares structural equation modeling (PLS-SEM. $2^{\text {nd }}$ ed.). Sage publications.

22. Hardy, C. (1996). Understanding power: Bringing about strategic change. British Journal of Management, 7(s1), S3-S16. https://doi. org/10.1111/j.1467-8551.1996.tb00144.x.

23. Janssen, O. (2005). The joint impact of perceived influence and supervisor supportiveness on employee innovative behaviour. Journal of occupational and organizational psychology, 78(4), 573-579. https://doi. org/10.1348/096317905X25823

24. Jong, J. \& Hartog, D. (2008). Innovative Work Behavior: Measurement and Validation (SCALES-Initiative Working paper H200820). Scientific AnaLysis of Entrepreneurship and SMEs - Netherlands Ministry of Economic Affairs. 
25. Kleysen, R. F. \& Street, C. T. (2001). Toward a multi-dimensional measure of individual innovative behavior. Journal of Intellectual Capital, 2(3), 284-296. https://doi.org/10.1108/EUM0000000005660

26. Kline, R. B. (2015). Principles and practice of structural equation modeling. Guilford publications.

27. Koudelková, P. \& Milichovský, F. (2015). Successful innovation by motivation. Business: Theory and Practice, 16(3), 223-230. https://doi.org/10.3846/ btp.2015.472

28. Kunz, J. \& Linder, S. (2015). With a view to make things better: individual characteristics and intentions to engage in management innovation. Journal of Management and Governance, 19(3), 525-556. https://doi.org/10.1007/ s10997-013-9280-7

29. Lahey, B. B. (1999). Introducción a la Psicología. McGraw Hill.

30. Lai, E. (2011). Motivation: A Literature Review (Research Report). Pearson. https://images.pearsonassessments.com/images/tmrs/Motivation_Review_ final.pdf

31. Larawan, L. A. (2011). Employee Innovativeness and Achievement motivation: A public and a private organization's experience. International Journal of Business and Management Studies, 3(2), 145-155.

32. Lo, H. Y. (2014). Quick response codes around us: Personality traits, attitudes toward innovation, and acceptance. Journal of Electronic Commerce Research, 15(1), 25-39.

33. McCabe, D. (2002). 'Waiting for dead men's shoes': Towards a cultural understanding of management innovation. Human Relations, 55(5), 505-536.

34. McClelland, D.(1961). The Achieving Society. D.Van Nostrand Company. https:// babel.hathitrust.org/cgi/pt?id=mdp.39015003646802\&view=1 up\&seq=7

35. McClelland, D. C. (1989). Estudio de la motivación humana. Narcea Ediciones.

36. McClelland, D. C. \& Burnham, D. H. (2003). Power is the great motivator. Harvard Business Review, 81(1), 117-126.

37. Monroy, A. y Rodríguez, G. S. (2012). Las teorías sobre la motivación y su aplicación a la actividad física y el deporte. Lecturas: Educación física y deportes, (164), 1-8.

38. Montero, R. (2016). Modelos de regresión lineal múltiple. Departamento de Economía Aplicada, Universidad de Granada.

39. Mura, M. \& Longo, M. (2013). Developing a tool for intellectual capital assessment: An individual-level perspective. Expert Systems, 30(5), 436-450. https://doi.org/10.1111/j.1468-0394.2012.00650. 
40. Pérez, J., Ceballos, G. y Cogco, A. (Coord.). (2014). Los retos de la politica industrial ante la reconfiguración espacial en México ( 1 a ed.). MAPorrúa.

41. Rogers, E. M. (2003). Diffusion of innovations ( $5^{\text {th }}$ ed.). Simon and Schuster.

42. Schumpeter, J. (1934). The Theory of Economic Development: An inquiry into profits, capital, credit, interest and the business cycle. Harvard University Press.

43. Shalley, C., Zhou, J. \& Oldham, G. (2004). The Effects of Personal and Contextual Characteristics on Creativity: Where Should We Go from Here? Journal of Management, 3(6), 933-958. https://doi.org/10.1016/j.jm.2004.06.007

44. Soriano, M. M. (2001). La motivación, pilar básico de todo tipo de esfuerzo. Proyecto social: Revista de relaciones laborales, (9), 163-184.

45. Valera, R. (2008). Innovación empresarial: arte y ciencia en la creación de empresas ( $3^{\mathrm{a}}$ ed.). Pearson.

46. Varadarajan, P. R. \& Jayachandran, S. (1999). Marketing strategy: an assessment of the state of the field and outlook. Journal of the academy of marketing science, 27(2), 120-143.

47. Xu, Y. (2011). A Social-Cognitive Perspective on Firm Innovation. Academy of Strategic Management Journal. Allied Business Academies, 7(1), 11-27.

48. Yuan, F. \& Woodman, R. (2010). Innovative behavior in the workplace: the role of performance and image outcome expectations. Academy of Management Journal, 53(2), 323-342.

49. Zhou, J. \& George, J. (2001). When job dissatisfaction leads to creativity: Encouraging the expression of voice. The Academy of Management Journal, 44(4), 682-696.

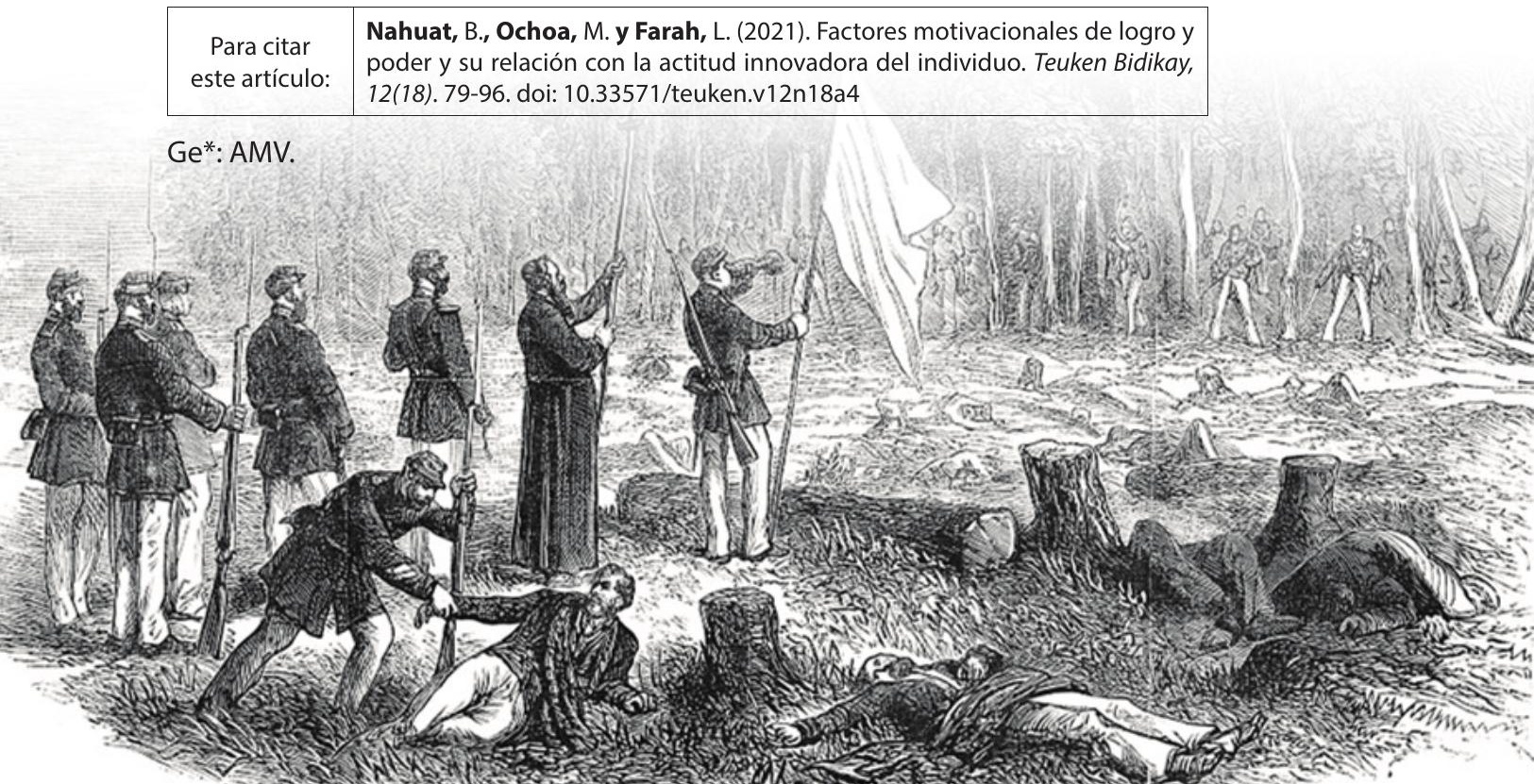

